\title{
Studies Toward The Synthesis of Pinnatoxins: The B,C,D-Dispiroketal Fragment
}

Chong-Dao Lu and Armen Zakarian*

\section{Supporting Information}

Experimental procedures, spectroscopic and analytical data for the new compounds described in this communication.

General Information. All reactions were carried out under an inert atmosphere of dry nitrogen in oven or flame-dried glassware. Proton magnetic resonance spectra were recorded at 300 and $500 \mathrm{MHz}$ on Varian Mercury and Varian Unity Inova spectrometers, respectively. Carbon magnetic resonance spectra were recorded at 75 MHz on a Varian Mercury spectrometer. Optical rotations were measured on a JASCO P-2000 polarimeter. High resolution mass spectral data were obtained by the Mass spectrometry laboratory at the Florida state University. Analytical thin-layer chromatography (TLC) was performed using pre-coated TLC plates with silica Gel 60 F254 (E. Merck no. 5715-7). Flash column chromatography was performed using 40-63 (m silica gel (Merck, Geduran, no. 11567-1) as the stationary phase. Tetrahydrofuran (THF) and ether were dried by distilling from sodium-benzophenone in a continuous still under an atmosphere of argon. Dichloromethane, di-iso-propylamine, triethylamine were distilled from calcium hydride in a continuous still under an atmosphere of argon.

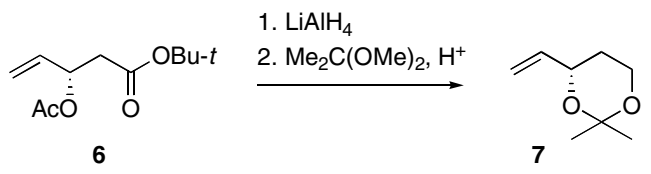

Acetonide 7. A solution of $(S)$-acetate $6^{1}(28.2 \mathrm{~g}, 131.6 \mathrm{mmol})$ in ether (100 $\left.\mathrm{mL}\right)$ was added to a suspension of $\mathrm{LiAlH}_{4}(7.5 \mathrm{~g}, 197.4 \mathrm{mmol})$ in ether $(160 \mathrm{~mL})$ at $0{ }^{\circ} \mathrm{C}$, and the mixture was allowed to warm to room temperature and stirred for another 3 . Solid $\mathrm{K}_{2} \mathrm{CO}_{3}$ and saturated solution of $\mathrm{MgSO}_{4}$ were added slowly and stirred for 1 h at room temperature. The white precipitate was filtered off and the solution was concentrated under reduced pressure to give $13.4 \mathrm{~g}$ of the crude diol, which was used in the next step without further purification.

A mixture of the crude diol (13.4 g) and $\mathrm{TsOH} \cdot \mathrm{H}_{2} \mathrm{O}(1.24 \mathrm{~g}, 6.5 \mathrm{mmol})$ in 2,2 dimethoxypropane (160 mL) was stirred overnight under room temperature. The reaction mixture was diluted with ether $(600 \mathrm{~mL})$, and washed with saturated aqueous $\mathrm{NaHCO}_{3}$, water, and brine. The organic layer was separated, dried over $\mathrm{MgSO}_{4}$, filtered and concentrated carefully. The residue was distilled under reduced pressure (61 ${ }^{\circ} \mathrm{C} / 20$ mmHg) to give acetonide $7(15.0 \mathrm{~g}, 105.3 \mathrm{mmol}, 80 \%$ for two steps) as colorless oil. $\left[\langle]_{\mathrm{D}}{ }^{23}+0.7^{\circ}\left(\mathrm{C} 1.0, \mathrm{CH}_{2} \mathrm{Cl}_{2}\right) ;{ }^{1} \mathrm{H} \mathrm{NMR}\left(300 \mathrm{MHz}, \mathrm{CDCl}_{3}\right) ;{ }^{\mathrm{TM}}(\mathrm{ppm}): 5.83\right.$ (septet, J=6.0 Hz,

\footnotetext{
${ }^{1}$ Tan, C.-H.; Holmes A. B. Chem. Eur. J. 2001, 7, 1845-1854
} 
$1 \mathrm{H}) ; 5.25\left(\mathrm{dd}, \mathrm{J}_{1}=18.0 \mathrm{~Hz}, \mathrm{~J}_{2}=1.2 \mathrm{~Hz}, 1 \mathrm{H}\right) ; 5.13\left(\mathrm{dd}, \mathrm{J}_{1}=18.0 \mathrm{~Hz}, \mathrm{~J}_{2}=1.2 \mathrm{~Hz}, 1 \mathrm{H}\right) ; 4.41-$ $4.34(\mathrm{~m} \mathrm{1H}), 4.01\left(\mathrm{dt}, \mathrm{J}_{\mathrm{t}}=12.0 \mathrm{~Hz}, \mathrm{~J}_{\mathrm{d}}=3.0 \mathrm{~Hz}, 1 \mathrm{H}\right) ; 3.85$ ( ddd, $\mathrm{J}_{1}=12.0 \mathrm{~Hz}, \mathrm{~J}_{2}=6.0 \mathrm{~Hz}$, $\left.\mathrm{J}_{3}=1.2 \mathrm{~Hz}, 1 \mathrm{H}\right) ; 1.72\left(\mathrm{dq}, \mathrm{J}_{\mathrm{q}}=12.0 \mathrm{~Hz}, \mathrm{~J}_{\mathrm{d}}=5.4 \mathrm{~Hz}, 1 \mathrm{H}\right) ; 1.53-1.39$ (m, $\left.1 \mathrm{H}\right) ; 1.49$ ( $\left.\mathrm{s}, 3 \mathrm{H}\right)$; $1.42(\mathrm{~s}, 3 \mathrm{H}) \cdot{ }^{13} \mathrm{C} \mathrm{NMR}\left(75 \mathrm{MHz}, \mathrm{CDCl}_{3}\right) ; \mathrm{TM}(\mathrm{ppm}): 138.7,115.4,98.3,70.0,59.6,31.0$, $29.9,19.2$.

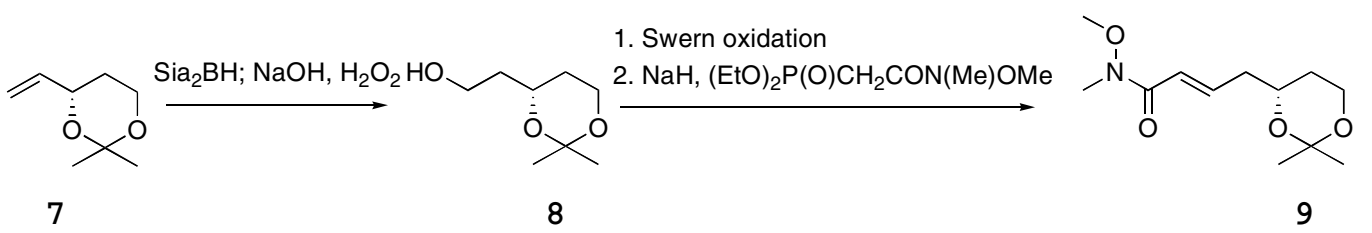

Weinreb amide 9. To a solution of $\mathrm{BH}_{3} \cdot \mathrm{SMe}_{2}(14.5 \mathrm{~mL}, 141.8 \mathrm{mmol})$ in $\mathrm{THF}$ (140 $\left.\mathrm{mL}\right)$ at 0 ${ }^{\circ} \mathrm{C}$ was added a solution of 2 -methyl-2-butene (30 mL, $283.6 \mathrm{mmol}$ ) in $\mathrm{THF}$ (140 $\mathrm{mL}$ ). After the mixture was stirred for 1 h at $0{ }^{\circ} \mathrm{C}$, a solution of the olefin 7 (10.0 $\mathrm{g}$, $70.3 \mathrm{mmol})$ in THF (70 mL) was added dropwise and stirring was continued for 2 h at 0 ${ }^{\circ} \mathrm{C} .6 \mathrm{~N} \mathrm{NaOH}(140 \mathrm{~mL}, 840 \mathrm{mmol})$ was slowly added followed by $30 \% \mathrm{H}_{2} \mathrm{O}_{2}(60 \mathrm{~mL})$. The mixture was vigorously stirred at room temperature for 30 min and then diluted with ether $(600 \mathrm{~mL})$. The organic phase was separated and washed with $\mathrm{H}_{2} \mathrm{O}$, $10 \%$ aqueous $\mathrm{NaHSO}_{3}$, saturated aqueous $\mathrm{NaHCO}_{3}$, brine, dried $\left(\mathrm{MgSO}_{4}\right)$, and filtered. The filtrate was concentrated in vacuo to afford crude alcohol 8 (10.2 g, 63.7 mmol, 91\%), which was used in the next step without further purification.

To a solution of oxalyl chloride (8.2 mL, 94 mmol) in $\mathrm{CH}_{2} \mathrm{Cl}_{2}$ (100 mL) was added a solution of DMSO (12 $\mathrm{mL}, 169 \mathrm{mmol})$ in $\mathrm{CH}_{2} \mathrm{Cl}_{2}(10 \mathrm{~mL})$ at -78 ${ }^{\circ} \mathrm{C}$ and the reaction mixture was stirred for $15 \mathrm{~min}$ at the same temperature. A solution of alcohol 8 (10.0 g, $62.4 \mathrm{mmol})$ in $\mathrm{CH}_{2} \mathrm{Cl}_{2}(20 \mathrm{~mL})$ was then added and the reaction mixture was stirred for further $20 \mathrm{~min}$ at $-78{ }^{\circ} \mathrm{C} . \mathrm{Et}_{3} \mathrm{~N}(35 \mathrm{ml}, 250 \mathrm{mmol})$ was then added and the reaction mixture was warmed to room temperature and stirred for further 1 h. The reaction mixture was directly filtered through a short pad of silica gel into the following reaction flask containing a solution of the Horner-Emmons reagent prepared from diethyl (N-methoxy-N-methylcarbamoylmethyl) phosphonate (25.7 $\mathrm{mL}, 124.8 \mathrm{mmol}) \mathrm{in} \mathrm{THF}$ \{the phosphonate was slowly added to a suspension of $\mathrm{NaH}(60 \%$ in $0 i 1,4.99 \mathrm{~g}, 124.8$ mmol) in THF $(200 \mathrm{~mL})$ at $0{ }^{\circ} \mathrm{C}$. The reaction mixture was allowed to warm to room temperature and stirred for $1 \mathrm{~h}$, and then cooled to $\left.0{ }^{\circ} \mathrm{C}\right\}$, and the silica gel was washed with $\mathrm{CH}_{2} \mathrm{Cl}_{2}(50 \mathrm{~mL})$. The solution was allowed to warm to room temperature and stirred for $30 \mathrm{~min}$, after which it was quenched with a saturated solution of $\mathrm{NH}_{4} \mathrm{Cl}$ $(100 \mathrm{~mL})$. The layers were separated and the aqueous layer was extracted with $\mathrm{CH}_{2} \mathrm{Cl}_{2}$, the combined organic extracts were washed with brine, dried with $\mathrm{MgSO}_{4}$, filtered, and concentrated under reduced pressure. Silica gel chromatography (ethyl acetate-hexanes $30 \%$ to 60\%) afforded vinyl amide 9 (13.4 g, $54.9 \mathrm{mmol}, 88 \% \mathrm{yield}) . \mathrm{l}_{\mathrm{D}}^{24}+14.2^{\circ}(\mathrm{c} 1.0$, $\left.\mathrm{CH}_{2} \mathrm{Cl}_{2}\right) ;{ }^{1} \mathrm{H} \mathrm{NMR}\left(300 \mathrm{MHz}, \mathrm{CDCl}_{3}\right) ;{ }^{\mathrm{TM}}(\mathrm{ppm}): 6.93$ (dt, $\left.\mathrm{J}_{\mathrm{d}}=15.6 \mathrm{~Hz}, \mathrm{~J}_{\mathrm{t}}=7.2 \mathrm{~Hz}, 1 \mathrm{H}\right) ; 6.47$ (d, $\mathrm{J}=15.6 \mathrm{~Hz}, 1 \mathrm{H}) ; 3.99$ (ddd, $\left.\mathrm{J}_{1}=13.2 \mathrm{~Hz}, \mathrm{~J}_{2}=12.0 \mathrm{~Hz}, \mathrm{~J}_{3}=3.0 \mathrm{~Hz}, 1 \mathrm{H}\right) ; 3.95\left(\mathrm{dt}, \mathrm{J}_{\mathrm{t}}=12.0 \mathrm{~Hz}\right.$, $\left.\mathrm{J}_{\mathrm{d}}=3.0 \mathrm{~Hz}, 1 \mathrm{H}\right) ; 3.82$ (ddd, $\left.\mathrm{J}_{1}=12.0 \mathrm{~Hz}, \mathrm{~J}_{2}=5.4 \mathrm{~Hz}, \mathrm{~J}_{3}=1.5 \mathrm{~Hz}, 1 \mathrm{H}\right) ; 3.70$ ( $3 \mathrm{H}) ; 2.49-2.30(\mathrm{~m}, 2 \mathrm{H}) ; 1.60\left(\mathrm{dq}, \mathrm{J}_{\mathrm{q}}=12.0 \mathrm{~Hz}, \mathrm{~J}_{\mathrm{d}}=5.4 \mathrm{~Hz}, 1 \mathrm{H}\right) ; 1.45$ (ddd, $\mathrm{J}_{1}=13.2 \mathrm{~Hz}$, 


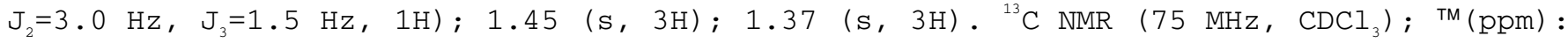
166.5, 142.7, 120.8, 98.2, 67.7,61.6,59.6, 39.3, 30.8, 29.7, 19.0. HRMS (ESI) Calcd for $\mathrm{C}_{12} \mathrm{H}_{21} \mathrm{NO}_{4} \mathrm{Na} \quad[\mathrm{M}+\mathrm{Na}] 266.1368$, found 266.1373 .

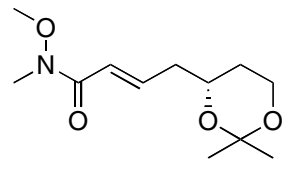

9

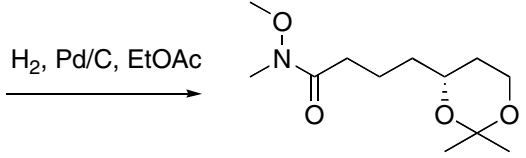

s9

Weinreb amide s9: A solution of amide 9 (13.4 g, $55.1 \mathrm{mmol}$ ) in ethyl acetate (100 mL) containing $10 \% \mathrm{Pd} / \mathrm{C}(650 \mathrm{mg})$ was stirred under $\mathrm{H}_{2}$ (1 atm) for 10 h at room temperature. The reaction mixture was filtered through silica gel and then concentrated under reduced pressure to give amide s9 (13.5 g, 55.0 mmol, 100\%). [〈] ${ }_{\mathrm{D}}^{24}$ $+10.0^{\circ}\left(\mathrm{c} 1.0, \mathrm{CH}_{2} \mathrm{Cl}_{2}\right) ;{ }^{1} \mathrm{H} \mathrm{NMR}\left(300 \mathrm{MHz}, \mathrm{CDCl}_{3}\right) ;{ }^{\mathrm{TM}}(\mathrm{ppm}): 3.94 \quad\left(\mathrm{dt}, \mathrm{J}_{\mathrm{t}}=12.0 \mathrm{~Hz}, \mathrm{~J}_{\mathrm{d}}=3.0 \mathrm{~Hz}\right.$, $1 \mathrm{H}) ; 3.88-3.77(\mathrm{~m}, 2 \mathrm{H}) ; 3.65(\mathrm{~s}, 3 \mathrm{H}) ; 3.16(\mathrm{~s}, 3 \mathrm{H}) ; 2.43(\mathrm{t}, \mathrm{J}=7.2 \mathrm{~Hz}, 2 \mathrm{H}) ; 1.77-1.39$ $(\mathrm{m}, 6 \mathrm{H}) ; 1.43(\mathrm{~s}, 3 \mathrm{H}) ; 1.36(\mathrm{~s}, 3 \mathrm{H}) \cdot{ }^{13} \mathrm{C} \operatorname{NMR}\left(75 \mathrm{MHz}, \mathrm{CDCl}_{3}\right) ;{ }^{\mathrm{TM}}(\mathrm{ppm}): 173.7$, 97.4, $68.0,60.6,59.3,35.4,31.0,30.6,29.4,19.6,18.6 . \mathrm{HRMS}_{(\mathrm{ESI})} \mathrm{Calcd}_{\mathrm{for}} \mathrm{C}_{12} \mathrm{H}_{23} \mathrm{NO}_{4} \mathrm{Na}$ $[\mathrm{M}+\mathrm{Na}] 268.1525$, found 268.1527 .

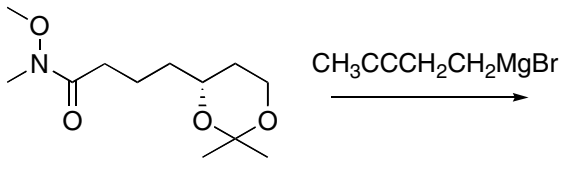

S9

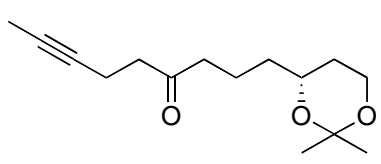

10

Ketone 10: To $3.6 \mathrm{~g}$ (150 mmol) of magnesium turnings covered with $20 \mathrm{~mL}$ THF was added $10 \mathrm{~mL}$ of a solution of 1 -bromo-3-pentyne (15 g, $100 \mathrm{mmol})$ in $160 \mathrm{~mL}$ THF. The suspension was heated to reflux and after the reaction was initiated, the solution of bromide was added to the reaction mixture at a rate sufficient to maintain a gentle reflux. After the addition was complete, the mixture was refluxed for further 1 h. The organomagnesium reagent solution was transferred via cannula into a solution of the amide s9 $(7.36 \mathrm{~g}, 30 \mathrm{mmol})$ in THF $200 \mathrm{~mL}$ at $0{ }^{\circ} \mathrm{C}$, and the reaction mixture was stirred for $1 \mathrm{~h}$ at $0{ }^{\circ} \mathrm{C}$ and $2 \mathrm{~h}$ at room temperature, and then quenched with $1 \mathrm{M}$ $\mathrm{NaH}_{2} \mathrm{PO}_{4}$. The aqueous phase was extracted with EtOAc, and the combined organic layers were washed with brine, dried over $\mathrm{MgSO}_{4}$, filtered, and concentrated under reduced pressure. Silica gel chromatography (10\% ethyl acetate-hexanes) afforded ketone 10 $(5.68 \mathrm{~g}, 22.5 \mathrm{mmol}, 75 \%) .\left[\langle]_{\mathrm{D}}^{24}-3.2^{\circ}\left(\mathrm{C} 1.0, \mathrm{CH}_{2} \mathrm{Cl}_{2}\right) ;{ }^{1} \mathrm{H} \mathrm{NMR}\left(300 \mathrm{MHz}_{\mathrm{C}} \mathrm{CDCl}{ }_{3}\right) ; \mathrm{TM}(\mathrm{ppm})\right.$ : $3.95\left(\mathrm{dt}, \mathrm{J}_{\mathrm{t}}=12.0 \mathrm{~Hz}, \mathrm{~J}_{\mathrm{d}}=3.0 \mathrm{~Hz}, 1 \mathrm{H}\right) ; 3.86-3.78(\mathrm{~m}, 2 \mathrm{H}) ; 2.58\left(\mathrm{dd}, \mathrm{J}_{1}=7.8 \mathrm{~Hz}, \mathrm{~J}_{2}=6.3 \mathrm{~Hz}\right.$, $2 \mathrm{H}) ; 2.44(t, J=7.2 \mathrm{~Hz}, 2 \mathrm{H}) ; 2.41-2.33(\mathrm{~m}, 2 \mathrm{H}) ; 1.74(\mathrm{t}, \mathrm{J}=2.7 \mathrm{~Hz}, 3 \mathrm{H}) ; 1.73-1.37(\mathrm{~m}$ ， $5 \mathrm{H}) ; 1.44(\mathrm{~s}, 3 \mathrm{H}) ; 1.36(\mathrm{~s}, 3 \mathrm{H}) \cdot{ }^{13} \mathrm{C} \mathrm{NMR}\left(75 \mathrm{MHz}, \mathrm{CDCl}_{3}\right)$; ${ }^{\mathrm{TM}}(\mathrm{ppm}): 208.8,97.9$, 77.5, $75.7,68.4,59.7,42.3,41.7,35.6,31.0,29.8,19.1,19.0,13.1,3.2$. HRMS (ESI) calcd for $\mathrm{C}_{15} \mathrm{H}_{24} \mathrm{O}_{3} \mathrm{Na}[\mathrm{M}+\mathrm{Na}] 275.1623$, found 275.1612. 


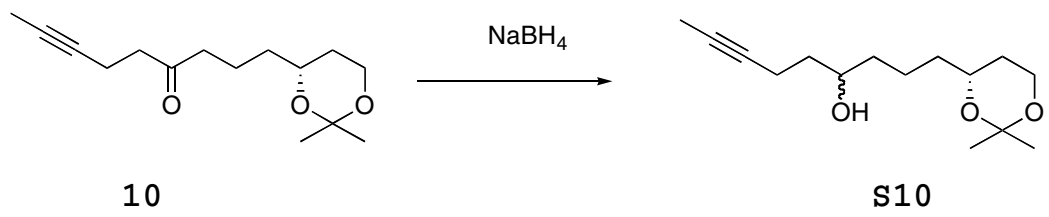

Alcohol s10. To a solution of the ketone 10 (2.52 g, $10 \mathrm{mmol}$ ) in methanol (20 mL) was added solid $\mathrm{NaBH}_{4}(378 \mathrm{mg}, 10 \mathrm{mmol})$ at $0{ }^{\circ} \mathrm{C}$. After the mixture was allowed to warm to room temperature while stirring, the solvent was removed under reduced pressure. The residue was diluted by ethyl acetate and washed with 1 N aqueous HCl, saturated aqueous $\mathrm{NaHCO}_{3}$, and brine, and dried over $\mathrm{MgSO}_{4}$ and filtered. The filtrate was concentrated in vacuo to afford crude alcohol s10 (2.47 g, 9.7 mmol, 97\%), which was used in the next step without further purification. A pair of isomers ( 1:1) ${ }^{1} \mathrm{H}$ NMR (300 $\left.\mathrm{MHz}, \mathrm{CDCl}_{3}\right)$; $\mathrm{TM}(\mathrm{ppm}): 3.95$ (dt, $\left.\mathrm{J}_{\mathrm{t}}=12.0 \mathrm{~Hz}, \mathrm{~J}_{\mathrm{d}}=3.0 \mathrm{~Hz}, 1 \mathrm{H}(\times 2)\right) ; 3.88-3.70 \quad(\mathrm{~m}$, $3 \mathrm{H}(\times 2)) ; 2.30-2.25(\mathrm{~m}, 2 \mathrm{H}(\times 2)) ; 1.91(\mathrm{~d}, \mathrm{~J}=4.2 \mathrm{~Hz}, 1 \mathrm{H},(\mathrm{OH})) ;[1.86(\mathrm{~d}, \mathrm{~J}=4.2 \mathrm{~Hz}, 1 \mathrm{H}$, $(\mathrm{OH}))] ; 1.77(\mathrm{t}, \mathrm{J}=2.7 \mathrm{~Hz}, 3 \mathrm{H}(\times 2)) ; 1.70-1.36(\mathrm{~m}, 10 \mathrm{H}(\times 2)) ; 1.44(\mathrm{~s}, 3 \mathrm{H}(\times 2)) ; 1.38(\mathrm{~s}$, $3 \mathrm{H}(\times 2)) \cdot{ }^{13} \mathrm{C} \mathrm{NMR}\left(75 \mathrm{MHz}, \mathrm{CDCl}_{3}\right) ;{ }^{\mathrm{TM}}(\mathrm{ppm}): 98.1,78.7,75.9,70.64(70.53), 68.77(68.65)$, $59.8,37.0(36.2), 36.01(35.96), 31.14(31.05), 29.8,20.97(20.79), 19.1,15.2,3.3$. HRMS (ESI) calcd for $\mathrm{C}_{15} \mathrm{H}_{27} \mathrm{O}_{3}[\mathrm{M}+\mathrm{H}]$ 255.1960, found 255.1958 .

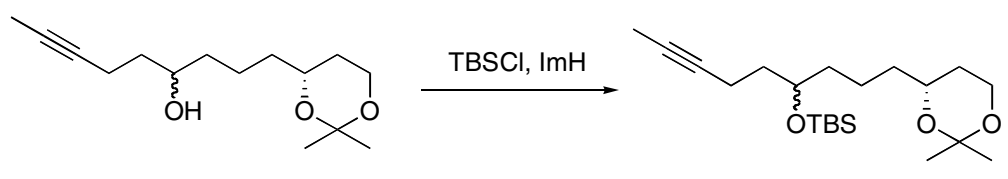

S10

11

TBS-Ether 11: To a solution of alcohol s10 (2.34 g, 9.2 mmol), imidazole (1.36 g, 20 mmol) and catalytic amount DMAP in DMF (20 ml) was added TBSCl (2.26 g, 15 mmol). The mixture was stirred for $3 \mathrm{~h}$ at $60{ }^{\circ} \mathrm{C}$ and cooled to room temperature, then DMF was removed under reduced pressure. The residue was diluted with dichloromethane, the solution was washed with brine and dried over $\mathrm{MgSO}_{4}$, filtered, and concentrated under reduced pressure. Silica gel chromatography (5\% ethyl acetate-hexanes) afforded TBSether 11 (3.35 g, $9.1 \mathrm{mmol}, 99 \%)$. A pair of isomers $(\sim 1: 1){ }^{1} \mathrm{H} \mathrm{NMR}\left(300 \mathrm{MHz}, \mathrm{CDCl}_{3}\right)$; $\mathrm{TM}(\mathrm{ppm}): 3.95$ (dt, $\left.\mathrm{J}_{\mathrm{t}}=12.0 \mathrm{~Hz}, \mathrm{~J}_{\mathrm{d}}=3.0 \mathrm{~Hz}, 1 \mathrm{H}\right) ; 3.86-3.72$ (m, 3H); $2.19-2.11$ (m, $\left.2 \mathrm{H}\right)$; 1.77 ( $t, \mathrm{~J}=2.4 \mathrm{~Hz}, 3 \mathrm{H}) ; 1.63-1.37(\mathrm{~m}, 10 \mathrm{H}) ; 1.49(\mathrm{~s}, 3 \mathrm{H}) ; 1.38(\mathrm{~s}, 3 \mathrm{H}) ; 0.88(\mathrm{~s}, 9 \mathrm{H})$; $0.06(\mathrm{~s}, 3 \mathrm{H}) ; 0.04(\mathrm{~s}, 3 \mathrm{H}) \cdot{ }^{13} \mathrm{C} \mathrm{NMR}\left(75 \mathrm{MHz}, \mathrm{CDCl}_{3}\right)$; ${ }^{\mathrm{TM}}(\mathrm{ppm}): 98.3,79.3,75.5,71.0$, $68.92(68.81), 60.1,37.03,36.79(36.69), 36.29(36.21), 31.14,30.1,20.6,19.3,18.2$, $15.0,3.6,-4.3,-4.4$. HRMS (ESI) calcd for $\mathrm{C}_{21} \mathrm{H}_{41} \mathrm{O}_{3} \mathrm{Si}[\mathrm{M}+\mathrm{H}] 369.2825$, found 369.2823 .

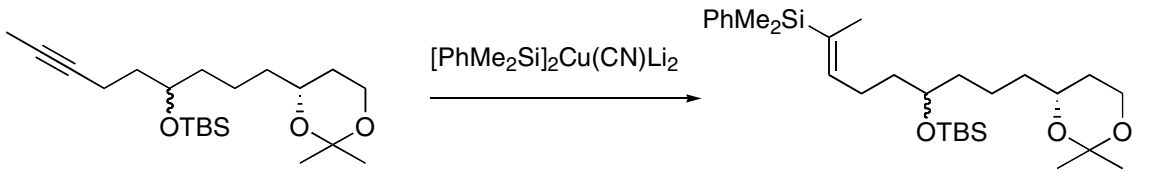


11

$\mathrm{S} 11$

Vinylsilane s11-1: To a mixture of CuCN (2.06 g, $23.0 \mathrm{mmol}$ ) and dry THF (10 ml) was added $\mathrm{PhMe}_{2} \mathrm{SiLi}$ (prepared according to Fleming's protocol, ${ }^{2} 0.9 \mathrm{M}$ in THF, $51.0 \mathrm{ml}$, $46.0 \mathrm{mmol})$ at $0{ }^{\circ} \mathrm{C}$ under a nitrogen atmosphere. The resultant red mixture was stirred at $0{ }^{\circ} \mathrm{C}$ for $20 \mathrm{~min}$, then cooled down to $-78{ }^{\circ} \mathrm{C}$. A solution of alkyne 11 (3.40 g, 9.2 mmol) in dry ether (50 ml) was added, and the mixture was stirred at $-78{ }^{\circ} \mathrm{C}$ for $2 \mathrm{~h}$ and at $0{ }^{\circ} \mathrm{C}$ for $15 \mathrm{~min}$. A saturated aqueous $\mathrm{NH}_{4} \mathrm{Cl}$-concentrated $\mathrm{NH}_{3}(9: 1 \mathrm{v} / \mathrm{v}, 40 \mathrm{ml})$ was added to quench the reaction, the mixture was stirred at $0{ }^{\circ} \mathrm{C}$ for $10 \mathrm{~min}$, then extracted with ethyl acetate $(2 \times 50 \mathrm{ml})$. Organic layers were washed with saturated aqueous $\mathrm{NH}_{4} \mathrm{Cl}$-concentrated $\mathrm{NH}_{3}(9: 1 \mathrm{v} / \mathrm{v}) \quad(20 \mathrm{ml})$ and brine (20 ml), dried over anhydrous $\mathrm{MgSO}_{4}$, filtered, and concentrated. Column chromatography (silica, 5\% ethyl acetate-hexanes) gave vinylsilane s11 (4.60 g, 9.1 mmol, 99\%). A pair of isomers $(\sim 1: 1){ }^{1} \mathrm{H} \mathrm{NMR}\left(300 \mathrm{MHz}, \mathrm{CDCl}_{3}\right) ; \delta(\mathrm{ppm}): 7.52-7.46(\mathrm{~m}, 2 \mathrm{H}) ; 7.35-7.32$ (m, 3H); 5.79 (dt, $\left.\mathrm{J}_{\mathrm{t}}=7.2 \mathrm{~Hz}, \mathrm{~J}_{\mathrm{d}}=1.2 \mathrm{~Hz}, 1 \mathrm{H}\right) ; 3.96$ (dt, $\mathrm{J}_{\mathrm{t}}=12.0 \mathrm{~Hz}, \mathrm{~J}_{\mathrm{d}}=3.0 \mathrm{~Hz}, 1 \mathrm{H}$ ); 3.83 (ddd, J $=11.4 \mathrm{~Hz}$, $\left.\mathrm{J}_{2}=5.4 \mathrm{~Hz}, \mathrm{~J}_{3}=1.8 \mathrm{~Hz}, 2 \mathrm{H}\right) ; 3.66-3.62(\mathrm{~m}, 1 \mathrm{H}) ; 2.26-2.02(\mathrm{~m}, 2 \mathrm{H}) ; 1.66(\mathrm{~s}, 3 \mathrm{H}) ; 1.71-$ $1.28(\mathrm{~m}, 10 \mathrm{H}) ; 1.45(\mathrm{~s}, 3 \mathrm{H}) ; 1.38(\mathrm{~s}, 3 \mathrm{H}) ; 0.89(\mathrm{~s}, 9 \mathrm{H}) ; 0.32(\mathrm{~s}, 6 \mathrm{H}) ; 0.04(\mathrm{~s}, 6 \mathrm{H})$. ${ }^{13} \mathrm{C} \mathrm{NMR}\left(75 \mathrm{MHz}, \mathrm{CDCl}_{3}\right) ; \delta(\mathrm{ppm}): 141.6,138.8,134.2,134.1,128.9,127.8,98.2,71.9$, $(68.9) 68.8,60.1,37.0(36.8), 36.7(36.6), 31.4,30.2,26.1,24.7, \quad(20.83) 20.77$, $19.38(19.35), 18.3,14.9,-3.28,-3.25,-4.2 . \operatorname{HRMS}(\mathrm{ESI}) \mathrm{calcd}$ for $\mathrm{C}_{29} \mathrm{H}_{53} \mathrm{O}_{3} \mathrm{Si} \mathrm{i}_{2}[\mathrm{M}+\mathrm{H}]$ 505.3533 , found 505.3529 .

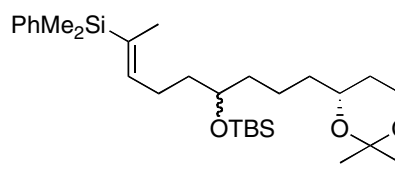

S11

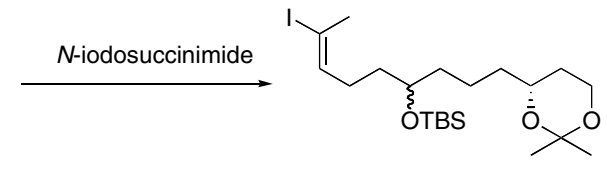

3

Iodoalkene 3: The vinylsilane s11 (5.04 g, 10.0 mol) was dissolved in 1,1,1,3,3,3hexafluoroisopropanol $(30 \mathrm{ml})$ and 2,6 -lutidine $(0.47 \mathrm{ml}, 4.0 \mathrm{mmol})$ was added. The solution was cooled to $0{ }^{\circ} \mathrm{C}$ and $N$-iodosuccinimide $(3.38 \mathrm{~g}, 15.0$ mmol) was added in one portion. After stirring for $5 \mathrm{~min}$, the reaction mixture was poured into a separatory funnel containing dichloromethane (150 ml) and saturated aqueous sodium sulfite $(50 \mathrm{ml})$. The organic layer was washed with saturated aqueous sodium bicarbonate $(20 \mathrm{ml})$ and brine $(20 \mathrm{ml})$. The combined aqueous layers were extracted with dichloromethane $(2 \times 20 \mathrm{ml})$. The combined organic layers were dried over sodium sulfate, filtered, and evaporated. The residue was purified by flash column chromatography (silica, 5\% ethyl acetate-hexanes) to yield iodoalkene 3 (4.72 g, 0.95 mmol, 95\%). A pair of isomers ( 1:1) ${ }^{1} \mathrm{H} \operatorname{NMR}\left(300 \mathrm{MHz}, \mathrm{CDCl}_{3}\right)$; ${ }^{\mathrm{TM}}(\mathrm{ppm}): 6.15$ (dt, $\mathrm{J}_{\mathrm{t}}=7.2$ $\left.\mathrm{Hz}, \mathrm{J}_{\mathrm{d}}=1.2 \mathrm{~Hz}, 1 \mathrm{H}\right) ; 3.96\left(\mathrm{dt}, \mathrm{J}_{\mathrm{t}}=12.0 \mathrm{~Hz}, \mathrm{~J}_{\mathrm{d}}=3.0 \mathrm{~Hz}, 1 \mathrm{H}\right) ; 3.82\left(\mathrm{ddd}, \mathrm{J}_{1}=11.4 \mathrm{~Hz}, \mathrm{~J}_{2}=5.4\right.$ $\left.\mathrm{Hz}, \mathrm{J}_{3}=1.8 \mathrm{~Hz}, 2 \mathrm{H}\right) ; 3.66-3.59(\mathrm{~m}, 1 \mathrm{H}) ; 2.36(\mathrm{~s}, 3 \mathrm{H}) ; 2.15-1.93(\mathrm{~m}, 2 \mathrm{H}) ; 1.63-1.23(\mathrm{~m}$ ， $\left.10 \mathrm{H}) ; 1.44(\mathrm{~s}, 3 \mathrm{H}) ; 1.38(\mathrm{~s}, 3 \mathrm{H}) ; 0.88(\mathrm{~s}, 9 \mathrm{H}) ; 0.04(\mathrm{~s}, 6 \mathrm{H}) .{ }^{13} \mathrm{C} \mathrm{NMR}_{(75} \mathrm{MHz} \mathrm{CDCl}_{3}\right)$; TM $(\mathrm{ppm}):$ 141.1, 98.0, 93.4, 71.4, (68.7)68.6, 59.9, 36.8, 36.6(36.5), (36.0)35.9,

2 Fleming, I.; Newton, T. W.; Roessler, F. J. Chem. Soc., Perkin Trans. 1 1981, 1, $2527-2532$. 
$31.3, \quad 30.0,27.4,(26.5) 25.9,20.7,19.3,18.1,-4.3,-4.4$. HRMS (ESI) calcd for $\mathrm{C}_{21} \mathrm{H}_{42} \mathrm{IO}_{3} \mathrm{Si}[\mathrm{M}+\mathrm{H}] \quad 497.1948$, found 497.2070 .

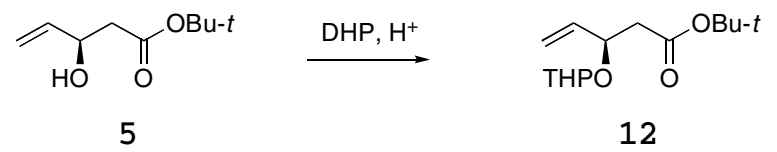

THP-Ether 12: To a solution of (R)-alcohol 5 (5.17 g, 30 mmol) and dihydropyran (5.47 $\mathrm{mL}, 60 \mathrm{mmol})$ in dichloromethane $(60 \mathrm{~mL})$ was added $\mathrm{TsOH} \cdot \mathrm{H}_{2} \mathrm{O}(57.1 \mathrm{mg}, 0.30 \mathrm{mmol})$ at room temperature. The reaction mixture was stirred for $2 \mathrm{~h}$ at room temperature and diluted with $50 \mathrm{~mL}$ dichloromethane and washed with saturated solution of sodium dicarbonate, then brine. The organic layer was dried over $\mathrm{MgSO}_{4}$, filtered and concentrated. The residue was purified by flash column chromatography (silica, 7\% ethyl acetate-hexanes) to yield THP-ether 12 (7.50 g, $29.3 \mathrm{mmol,} \mathrm{98 \% ).} \mathrm{A} \mathrm{pair} \mathrm{of}$ isomers: ${ }^{1} \mathrm{H} \mathrm{NMR}\left(300 \mathrm{MHz}, \mathrm{CDCl}_{3}\right) ;{ }^{\mathrm{TM}}(\mathrm{ppm}):\left[5.92\right.$ (ddd, $\mathrm{J}_{1}=17.4 \mathrm{~Hz}, \mathrm{~J}_{2}=10.8 \mathrm{~Hz}, \mathrm{~J}_{3}=6.6 \mathrm{~Hz}$, $1 \mathrm{H})] ; 5.66\left(\mathrm{ddd}, \mathrm{J}_{1}=18.0 \mathrm{~Hz}, \mathrm{~J}_{2}=10.2 \mathrm{~Hz}, \mathrm{~J}_{3}=7.8 \mathrm{~Hz}, 1 \mathrm{H}\right) ; 5.31-5.20$ (m, 2H); [5.12 (dt, $\left.\left.\mathrm{J}_{\mathrm{t}}=6.0 \mathrm{~Hz}, \mathrm{~J}_{\mathrm{d}}=1.2 \mathrm{~Hz}, 1 \mathrm{H}\right)\right] ;[4.75$ ( $\mathrm{t}, \mathrm{J}=4.8 \mathrm{~Hz}, 1 \mathrm{H}] ; 4.71$ ( $\mathrm{t}, \mathrm{J}=3.6 \mathrm{~Hz}, 1 \mathrm{H} ; 4.60-4.43$ $(\mathrm{m}, 1 \mathrm{H}) ;[4.60-4.43(\mathrm{~m}, 1 \mathrm{H})] ; 3.95-3.84(\mathrm{~m} \mathrm{1H}) ;[3.95-3.84(\mathrm{~m} 1 \mathrm{H})] ; 3.54-3.43(\mathrm{~m}, 1 \mathrm{H})$; $[3.54-3.43(\mathrm{~m}, 1 \mathrm{H})] ; 2.56\left(\mathrm{dt}, \mathrm{J}_{\mathrm{t}}=13.8 \mathrm{~Hz}, \mathrm{~J}_{\mathrm{d}}=7.8 \mathrm{~Hz}, 1 \mathrm{H}\right) ;\left[2.56\right.$ (dt, $\mathrm{J}_{\mathrm{t}}=13.8 \mathrm{~Hz}$, $\left.\left.\mathrm{J}_{\mathrm{d}}=7.8 \mathrm{~Hz}, 1 \mathrm{H}\right)\right] ; 2.40\left(\mathrm{dd}, \mathrm{J}_{1}=14.4 \mathrm{~Hz}, \mathrm{~J}_{2}=6.0 \mathrm{~Hz}, 1 \mathrm{H}\right) ;\left[2.40\left(\mathrm{dd}, \mathrm{J}_{1}=15.0 \mathrm{~Hz}, \mathrm{~J}_{2}=4.8 \mathrm{~Hz}\right.\right.$, $1 \mathrm{H})] ; 1.87-1.44(\mathrm{~m}, 8 \mathrm{H}) ; 1.45(\mathrm{~s}, 9 \mathrm{H}) ;[1.44(\mathrm{~s}, 9 \mathrm{H})]$. HRMS (ESI) calcd for $\mathrm{C}_{14} \mathrm{H}_{24} \mathrm{O}_{4} \mathrm{Na}$ $[\mathrm{M}+\mathrm{Na}] 279.1572$, found 279.1567 .

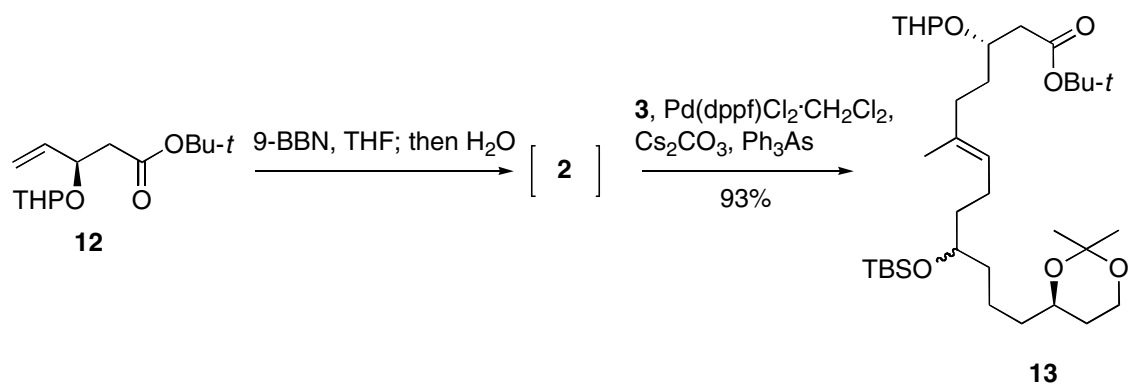

Olefin 13: To a solution of 12 (5.12 $\mathrm{g}, 20 \mathrm{mmol})$ in THF (50 mL) was added 9 -BBN dimer $(3.66 \mathrm{~g}, 15 \mathrm{mmol})$. After the resulting mixture was stirred at room temperature for 1 h. TLC analysis indicated the complete consumption of the starting olefin 12 . In a separate flask containing vinyl iodide $3(5.0 \mathrm{~g}, 10.0 \mathrm{mmol}),(\mathrm{dppf}) \mathrm{PdCl}_{2} \cdot \mathrm{CH}_{2} \mathrm{Cl}_{2}(0.816$ g, $1.0 \mathrm{mmol}), \mathrm{AsPh}_{3}(0.306 \mathrm{~g}, 1.0 \mathrm{mmol})$, and $\mathrm{Cs}_{2} \mathrm{CO}_{3}(4.89 \mathrm{~g}, 15.0$ mmol) was added degassed DMF (35 mL). Water $(5 \mathrm{~mL})$ was added to the borane solution and stirring was continued for $10 \mathrm{~min}$ to quench the excess 9 -BBN-H. Then, the solution of the alkylborane was added rapidly to the vigorously stirred solution containing the iodoolefin. After $2 \mathrm{~h}$, the reaction mixture was diluted with ethyl acetate (300 mL), washed with water $(150 \mathrm{~mL})$ and brine $(100 \mathrm{~mL})$, dried over $\mathrm{MgSO}_{4}$, filtered, and concentrated in vacuo. Column chromatography on silica gel (10\% ethyl acetate-hexanes) provided olefin $13(5.85 \mathrm{~g}, 9.3 \mathrm{mmol}, 93 \%)$. Mixture of isomers: ${ }^{1} \mathrm{H} \mathrm{NMR}$ (300 MHz, 
$\left.\mathrm{CDCl}_{3}\right) ;{ }^{\mathrm{TM}}(\mathrm{ppm}): 5.11(\mathrm{t}, \mathrm{J}=7.2 \mathrm{~Hz}, 1 \mathrm{H}) ; 4.71-4.66(\mathrm{~m}, 1 \mathrm{H}) ; 4.03-3.78(\mathrm{~m}, 5 \mathrm{H}) ; 3.65-$ $3.60(\mathrm{~m}, 1 \mathrm{H}) ; 3.51-3.43(\mathrm{~m}, 1 \mathrm{H}) ; 2.67\left(\mathrm{dd}, \mathrm{J}_{1}=15.0 \mathrm{~Hz}, \mathrm{~J}_{2}=6.0 \mathrm{~Hz}, 1 \mathrm{H}\right) ; 2.43-2.33(\mathrm{~m}$, $2 \mathrm{H}) ; 2.04-1.24(\mathrm{~m}, 21 \mathrm{H}) ; 1.58(\mathrm{~s}, 3 \mathrm{H}) ; 1.45(\mathrm{~s}, 12 \mathrm{H}) ; 1.38(\mathrm{~s}, 3 \mathrm{H}) ; 0.88(\mathrm{~s}, 9 \mathrm{H})$; $\left.0.03(\mathrm{~s}, 6 \mathrm{H}) \cdot{ }^{13} \mathrm{C} \mathrm{NMR} \mathrm{(75} \mathrm{MHz,} \mathrm{CDCl}_{3}\right) ;{ }^{\mathrm{TM}}(\mathrm{ppm}): 170.7,(134.5) 134.3,124.6,(98.4) 97.9$, $(80.0) 79.9,74.1,71.8,(68.6) 68.5,(62.6) 62.3,59.8,42.1,41.7,36.8,36.6,36.5$, $35.0,32.3,31.2,30.9,29.9,28.0,25.8,19.1,18.0,15.8,15.8,-4.5$. HRMS (ESI) calcd for $\mathrm{C}_{35} \mathrm{H}_{66} \mathrm{O}_{7} \mathrm{SiNa}[\mathrm{M}+\mathrm{Na}] 649.4476$, found 649.4476 .

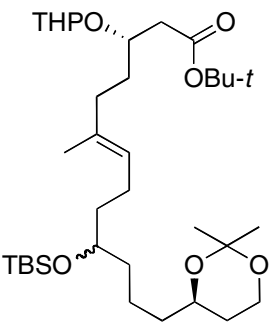

13

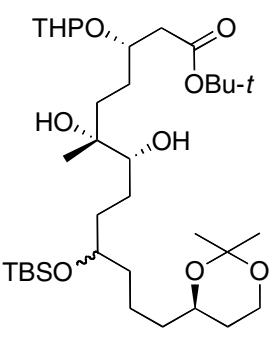

S13-1

Diol s13-1: Olefin $13(5.75 \mathrm{~g}, 9.17 \mathrm{mmol})$, potassium ferricyanate (9.06 g (27.5 mmol), potassium carbonate $(3.80 \mathrm{~g}, 27.5 \mathrm{mmol})$, methanesulfonamide (872 mg, $9.17 \mathrm{mmol})$, and (DHQD) ${ }_{2}$ PHAL $(358 \mathrm{mg}, 0.46 \mathrm{mmol})$ were combined in 1:1 mixture of tert-butanol and water $(100 \mathrm{~mL})$ and stirred at $0{ }^{\circ} \mathrm{C}$ for $30 \mathrm{~min}$. Potassium osmate dihydrate (34 mg, $0.092 \mathrm{mmol}$ ) was then added and the mixture stirred at $0{ }^{\circ} \mathrm{C}$ for $48 \mathrm{~h}$. The reaction was quenched with saturated aqueous $\mathrm{Na}_{2} \mathrm{~S}_{2} \mathrm{O}_{3}$, stirred at room temperature for 30 min and extracted three times with ethyl acetate. The combined organic layers was dried over $\mathrm{MgSO}_{4}$, filtered, and concentrated. The residue was purified by flash column chromatography (silica, 30\% to 50\% ethyl acetate-hexanes) to yield diol s13-1 (5.80 g, $8.80 \mathrm{mmol}, 96 \%)$ and recovered olefin $13100 \mathrm{mg}(0.16 \mathrm{mmol}, 2 \%)$. Mixture of isomers: ${ }^{1} \mathrm{H} \quad \mathrm{NMR}$ (300 MHz, $\left.\mathrm{CDCl}_{3}\right) ;{ }^{\mathrm{TM}}(\mathrm{ppm}): 4.72-4.61$ (m, $\left.1 \mathrm{H}\right) ; 4.18-3.26$ (m, 8H); 2.72-2.31 (m, $3 \mathrm{H}) ; 1.85-1.12(\mathrm{~m}, 21 \mathrm{H}) ; 1.45(\mathrm{~s}, 12 \mathrm{H}) ; 1.38(\mathrm{~s}, 3 \mathrm{H}) ; 1.08(\mathrm{~s}, 3 \mathrm{H}) ; 0.89-0.88(\mathrm{~m}, 9 \mathrm{H})$; 0.06-0.04 (m, 6H). $\left.{ }^{13} \mathrm{C} \mathrm{NMR} \mathrm{(75} \mathrm{MHz}, \mathrm{CDCl}_{3}\right) ;($ selected peaks) TM (ppm): 170.9, 98.0, 80.2, $74.3,72.4,68.5,59.9,41.7,36.4,33.8,31.2,29.9,28.0,25.8,19.2,18.0,-4.5$. HRMS (ESI) calcd for $\mathrm{C}_{35} \mathrm{H}_{68} \mathrm{O}_{9} \mathrm{SiNa}$ [M+Na] 683.4530, found 683.4525.
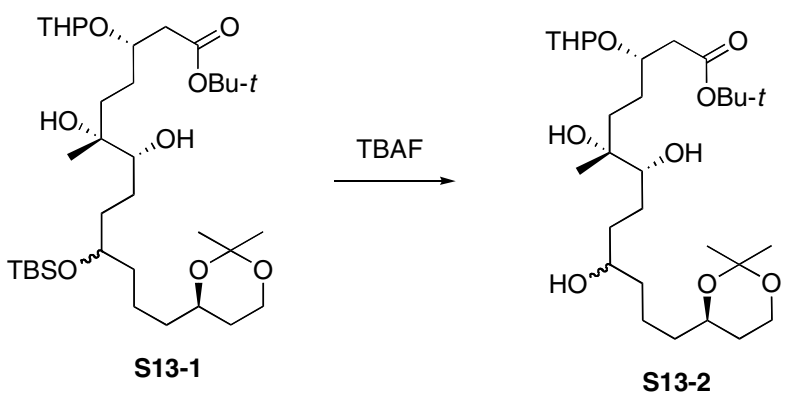

Triol s13-2: To a solution of the diol s13-1 (1.5 g, $2.27 \mathrm{mmol}$ ) in THF (25 mL) was added $\mathrm{TBAF} \cdot 3 \mathrm{H}_{2} \mathrm{O}(1.43 \mathrm{~g}, 4.54 \mathrm{mmol})$. The mixture was stirred for $8 \mathrm{~h}$ at room 
temperature, then diluted with ethyl acetate and washed with brine. The organic layer was dried over $\mathrm{MgSO}_{4}$, filtered, and concentrated. The residue was purified by flash column chromatography (silica, 70\% ethyl acetate-hexanes to 5\% methanol-ethyl acetate) to yield triol s13-2 (1.22 g, $2.22 \mathrm{mmol}, 98 \%)$. Mixture of isomers: ${ }^{1} \mathrm{H} \mathrm{NMR}$ (300 MHz, $\left.\mathrm{CDCl}_{3}\right) ; \mathrm{TM}(\mathrm{ppm}): 4.71-4.61(\mathrm{~m}, 1 \mathrm{H}) ; 4.24-3.77(\mathrm{~m}, 5 \mathrm{H}) ; 3.70-3.37$ (m, 3H); $3.02-2.28(\mathrm{~m}$, $4 \mathrm{H}) ; 1.82-1.26(\mathrm{~m}, 21 \mathrm{H}) ; 1.43(\mathrm{~s}, 12 \mathrm{H}) ; 1.36(\mathrm{~s}, 3 \mathrm{H}) ; 1.07(\mathrm{~s}, 3 \mathrm{H}) .{ }^{13} \mathrm{C} \mathrm{NMR}(75 \mathrm{MHz}$, $\left.\mathrm{CDCl}_{3}\right)$; (selected peaks) TM (ppm): 171.1, 98.1, 80.4, 74.3, 74.2, 71.1, 68.9, 68.8, 62.4, 59.9, 41.7, 36.3, 30.9, 29.9, 28.1, 25.3, 19.2. HRMS (ESI) calcd for $\mathrm{C}_{25} \mathrm{H}_{55} \mathrm{O}_{9}$ $[\mathrm{M}+\mathrm{H}] \quad 547.3846$, found 547.3833 .

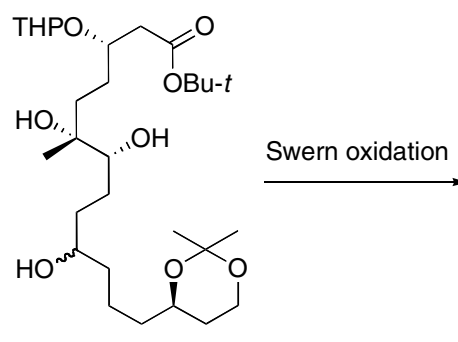

S13-2

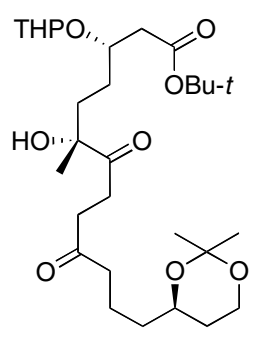

14

Diketone 14: To a solution of oxalyl chloride $(0.79 \mathrm{~mL}, 9.1 \mathrm{mmol})$ in dichloromethane $(40 \mathrm{~mL})$ at $-78{ }^{\circ} \mathrm{C}$ was added DMSO $(1.3 \mathrm{~mL}, 18.2 \mathrm{mmol})$ in dichloromethane (5 mL) dropwise. The reaction mixture was stirred for $15 \mathrm{~min}$ at $-78^{\circ} \mathrm{C}$, triol s13-2 (990 mg, $1.81 \mathrm{mmol})$ in dichloromethane (5 $\mathrm{mL})$ was added and the mixture stirred for additional $20 \mathrm{~min}$. Triethylamine $(3.8 \mathrm{~mL}, 27.2 \mathrm{mmol})$ was added and the reaction was warmed to room temperature and then stirred for $1 \mathrm{~h}$. Dichloromethane and water were added, and the organic layer was separated and washed with brine, dried over MgSO4, filtered, and concentrated. The residue was purified by flash column chromatography (silica, $40 \%$ ethyl acetate-hexanes) to yield diketone 14 (690 mg, $1.27 \mathrm{mmol}, 70 \%)$.

A pair of isomers: ${ }^{1} \mathrm{H} \mathrm{NMR}\left(300 \mathrm{MHz}, \mathrm{CDCl}_{3}\right)$; ${ }^{\mathrm{TM}}(\mathrm{ppm}): 4.71-4.60$ (m, 1H); 4.13-3.70 (m, $5 \mathrm{H}) ; 3.51-3.41(\mathrm{~m}, 1 \mathrm{H}) ; 2.92-2.60(\mathrm{~m}, 4 \mathrm{H}) ; 2.51-2.28(\mathrm{~m}, 4 \mathrm{H}) ; 1.89-1.16(\mathrm{~m}$ ，16H); $1.44(\mathrm{~s}, 12 \mathrm{H}) ; 1.38(\mathrm{~s}, 3 \mathrm{H}) ; 1.37(\mathrm{~s}, 3 \mathrm{H}) \cdot{ }^{13} \mathrm{C} \mathrm{NMR}\left(75 \mathrm{MHz}, \mathrm{CDCl}_{3}\right)$; (selected peaks) Tм $(\mathrm{ppm}): 213.6,208.8,170.7,98.1(97.9), \quad(80.5) 80.3, \quad(78.6) 78.5,73.8,68.8,62.5$, $59.9,42.4,41.6,35.9,35.8,34.5,31.1,30.9,29.9,28.0,27.4,25.6,25.4,19.6$, 19.3, 19.2. HRMS (ESI) calcd for $\mathrm{C}_{29} \mathrm{H}_{50} \mathrm{O}_{9} \mathrm{Na}[\mathrm{M}+\mathrm{Na}] 565.3353$, found 565.3356.

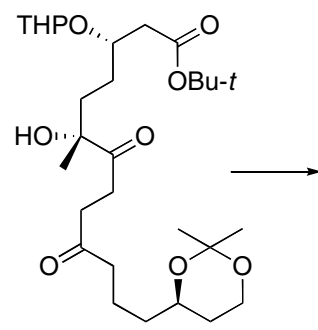

14

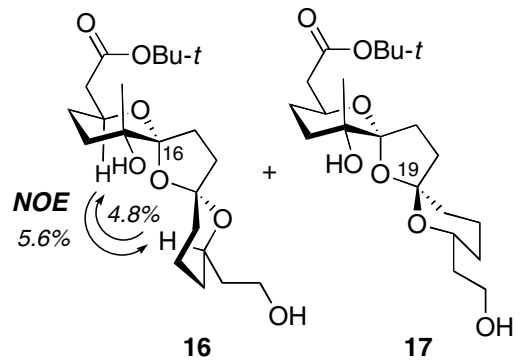

16
17 
Bisspiroketals 16 and 17: To a solution of the diketone $14(1.50 \mathrm{~g}, 2.76 \mathrm{mmol})$ in methanol (30 $\mathrm{mL})$ was added CSA $(64 \mathrm{mg}, 0.276 \mathrm{mmol})$. The mixture was stirred for $10 \mathrm{~h}$ at room temperature and methanol was removed under reduced pressure. Cyclohexane (110 $\mathrm{mL}$ ) was added and the mixture was stirred at room temperature for $48 \mathrm{~h}$. The reaction was quenched by saturated solution of $\mathrm{NaHCO}_{3}$ and the mixture was extracted with ethyl acetate. The combined organic layers were dried with $\mathrm{Na}_{2} \mathrm{SO}_{4}$, filtered, and concentrated. The residue was purified by flash column chromatography (silica, 30\% to 100\% ethyl acetate-hexanes) to yield desired bisspiroketal 16 (less polar one on TLC, $0.860 \mathrm{~g}, 2.15 \mathrm{mmol}, 78 \%)$, a mixture of partially ketalized by-products (173 $\mathrm{mg})$, and undesired isomer 17 (C19 epimer, the polar one on TLC, $90 \mathrm{mg}, 8.1 \%$ ). The mixture of the by-products and the undesired isomer $17(263 \mathrm{mg})$ were resubjected to equilibration according to the procedure described above to provide additional bisspiroketal 16 (106 mg, $0.265 \mathrm{mmol}, 9.5 \%)$, bringing the total yield of 16 to 0.966 g $(2.41 \mathrm{mmol}, 88 \%)$.

16: $\left[\langle]_{\mathrm{D}}{ }^{24}-8.1^{\circ}\left(\mathrm{C} 1.0, \mathrm{CH}_{2} \mathrm{Cl}_{2}\right) ;{ }^{1} \mathrm{H} \mathrm{NMR}\left(300 \mathrm{MHz}, \mathrm{C}_{6} \mathrm{D}_{6}\right) ; \mathrm{TM}^{\mathrm{TMpm}}\right): 4.49-4.39$ (m, $\left.1 \mathrm{H}\right) ; 4.27$ (tt, $\left.\mathrm{J}_{1}=10.2 \mathrm{~Hz}, \mathrm{~J}_{2}=3.0 \mathrm{~Hz}, 1 \mathrm{H}\right) ; 4.04-3.87(\mathrm{~m}, 2 \mathrm{H}) ; 3.51-3.49$ (br, 1H); 2.89 (dd, $\left.\mathrm{J}_{1}=14.4 \mathrm{~Hz}, \mathrm{~J}_{2}=5.4 \mathrm{~Hz}, 1 \mathrm{H}\right) ; 2.31\left(\mathrm{dd}, \mathrm{J}_{1}=14.4 \mathrm{~Hz}, \mathrm{~J}_{2}=8.4 \mathrm{~Hz}, 1 \mathrm{H}\right) ; 2.21-2.05(\mathrm{~m}, 2 \mathrm{H})$; $1.90\left(\mathrm{dt}, \mathrm{J}_{\mathrm{t}}=13.2 \mathrm{~Hz}, \mathrm{~J}_{\mathrm{d}}=4.2 \mathrm{~Hz}, 1 \mathrm{H}\right) ; 1.80-1.50(\mathrm{~m}, 7 \mathrm{H}) ; 1.45(\mathrm{~s}, 9 \mathrm{H}) ; 1.37$ (s, 3H); 1.42-1.02 (m, 7H). ${ }^{13} \mathrm{C}$ NMR $\left(75 \mathrm{MHz}, \mathrm{C}_{6} \mathrm{D}_{6}\right)$; ${ }^{\mathrm{TM}}(\mathrm{ppm}): 171.4,111.4,108.3,80.4,72.1$, $69.6,68.1,60.2,42.3,39.0,37.9,36.0,34.8,31.3,30.8,30.2,28.1,21.5,20.0$. HRMS (ESI) calcd for $\mathrm{C}_{21} \mathrm{H}_{36} \mathrm{O}_{7} \mathrm{Na}$ [M+Na] 423.2359, found 423.2349 .

17: $\left[\langle]_{\mathrm{D}}{ }^{24}+15.8^{\circ}\left(\mathrm{C} 1.0, \mathrm{CH}_{2} \mathrm{Cl}_{2}\right) ;{ }^{1} \mathrm{H} \mathrm{NMR}\left(300 \mathrm{MHz}, \mathrm{C}_{6} \mathrm{D}_{6}\right) ; \mathrm{TM}(\mathrm{ppm}): 4.47\right.$ (hep, J=4.2 $\mathrm{Hz}$, $1 \mathrm{H},) ; 3.67-3.61(\mathrm{~m}, 1 \mathrm{H}) ; 3.55\left(\mathrm{dt}, \mathrm{J}_{\mathrm{d}}=10.2 \mathrm{~Hz}, \mathrm{~J}_{\mathrm{t}}=5.4 \mathrm{~Hz}, 1 \mathrm{H}\right) ; 3.39$ (tt, $\mathrm{J}_{1}=10.2 \mathrm{~Hz}$, $\left.\mathrm{J}_{2}=3.0 \mathrm{~Hz}, 1 \mathrm{H}\right) ; 3.22-3.18(\mathrm{br}, 1 \mathrm{H}) ; 2.47-2.33(\mathrm{~m}, 2 \mathrm{H}) ; 2.14-2.00(\mathrm{~m}, 3 \mathrm{H}) ; 1.99-1.86$ $(\mathrm{m}, 3 \mathrm{H}) ; 1.75-1.54(\mathrm{~m}, 4 \mathrm{H}) ; 1.52-0.80(\mathrm{~m}, 7 \mathrm{H}) ; 1.39(\mathrm{~s}, 9 \mathrm{H}) ; 1.32(\mathrm{~s}, 3 \mathrm{H}) .{ }^{13} \mathrm{C}$ NMR $(75$ $\left.\mathrm{MHz}, \mathrm{C}_{6} \mathrm{D}_{6}\right)$; тм $(\mathrm{ppm}): 170.2,111.5,109.2,79.6,73.1,68.9,67.6,59.8,42.2,39.1$, $35.9,35.4,31.3,30.5,30.15,30.07,28.1,21.8,21.7$. HRMS (ESI) calcd for $\mathrm{C}_{21} \mathrm{H}_{36} \mathrm{O}_{7} \mathrm{Na}$ $[\mathrm{M}+\mathrm{Na}] 423.2359$, found 423.2366 .

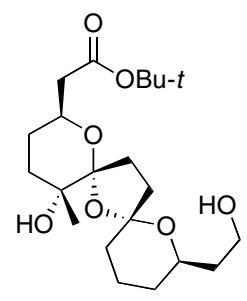

16

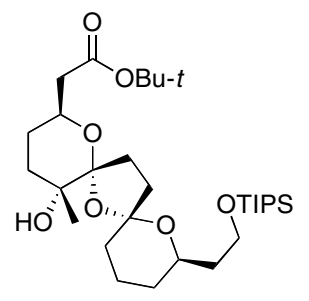

S16-1

TIPS-Ether S16-1: To a solution of the bisspiroketal $16(0.240 \mathrm{~g}, 0.60 \mathrm{mmol})$, imidazole (82 mg, $1.2 \mathrm{mmol})$ and DMAP $(7 \mathrm{mg}, 0.056 \mathrm{mmol})$ in dichloromethane $(2.0 \mathrm{~mL})$ was added TIPSCl $(0.19 \mathrm{~mL}, 0.90 \mathrm{mmol})$. The reaction mixture was stirred for $24 \mathrm{~h}$ at room temperature and then directly subjected to flash column chromatography (silica, 15\% ethyl acetate-hexanes) to yield TIPS-ether s16-1 (0.332 g, $0.596 \mathrm{mmol}, 99 \%)$. [ $]_{\mathrm{D}}^{24}$ $+1.0^{\circ}\left(\mathrm{C} 1.0, \mathrm{CH}_{2} \mathrm{Cl}_{2}\right) ;{ }^{1} \mathrm{H} \operatorname{NMR}\left(300 \mathrm{MHz}, \mathrm{C}_{6} \mathrm{D}_{6}\right) ;{ }^{\mathrm{TM}}(\mathrm{ppm}): 4.40-4.32(\mathrm{~m} ， 1 \mathrm{H}) ; 4.14-3.92$ (m， 
$3 \mathrm{H}) ; 2.68\left(\mathrm{dd}, \mathrm{J}_{1}=14.4 \mathrm{~Hz}, \mathrm{~J}_{2}=4.8 \mathrm{~Hz}, 1 \mathrm{H}\right) ; 2.39\left(\mathrm{dd}, \mathrm{J}_{1}=14.4 \mathrm{~Hz}, \mathrm{~J}_{2}=8.1 \mathrm{~Hz}, 1 \mathrm{H}\right) ; 2.30-$ $2.03(\mathrm{~m}, 3 \mathrm{H}) ; 1.98-1.78(\mathrm{~m}, 3 \mathrm{H}) ; 1.72-0.90(\mathrm{~m} \mathrm{10H}) ; 1.41(\mathrm{~s}, 9 \mathrm{H}) ; 1.31(\mathrm{~s}, 3 \mathrm{H}) ; 1.17$ $(\mathrm{s}, 21 \mathrm{H}) \cdot{ }^{13} \mathrm{C} \mathrm{NMR}\left(75 \mathrm{MHz}, \mathrm{C}_{6} \mathrm{D}_{6}\right) ;{ }^{\mathrm{TM}}(\mathrm{ppm}): 170.0,111.3,108.4,79.8,70.6,69.7,68.1$, $61.1,42.3,40.4,37.9,36.2,34.9,31.3,31.0,30.0,28.2,21.5,20.2,18.4,12.4$. HRMS (ESI) calcd for $\mathrm{C}_{30} \mathrm{H}_{56} \mathrm{O}_{7} \mathrm{SiNa}$ [M+Na] 579.3693, found 579.3687 .

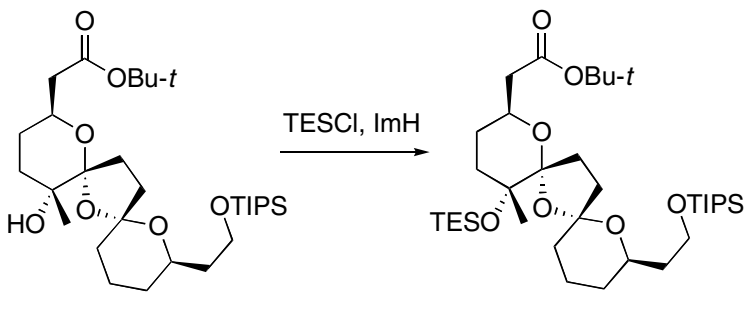

S16-1

S16-2

TES-Ether s16-2: To a solution of the TIPS-ether s16-1 (0.126 g, 0.226 mmol), imidazole (62 mg, $0.904 \mathrm{mmol})$ and DMAP (28 mg, $0.226 \mathrm{mmol})$ in DMF (0.5 mL) was added TESCl $(0.114 \mathrm{~mL}, 0.678 \mathrm{mmol})$. The reaction mixture was stirred for $48 \mathrm{~h}$ at $40^{\circ} \mathrm{C}$ and then directly subjected to flash column chromatography (silica, 10\% ethyl acetatehexanes) to yield TES-ether s16-2 (0.150 g, $0.224 \mathrm{mmol}, 99 \%) .\left[\langle]_{\mathrm{D}}^{24}+6.7^{\circ}(\mathrm{C} 1.0\right.$, $\left.\mathrm{CH}_{2} \mathrm{Cl}_{2}\right) ;{ }^{1} \mathrm{H}$ NMR $\left(300 \mathrm{MHz}, \mathrm{C}_{6} \mathrm{D}_{6}\right) ;{ }^{\mathrm{TM}}(\mathrm{ppm}): 4.57-4.50$ (m，1H);4.22-4.08 (m, 2H);4.00 (dt， $\left.\mathrm{J}_{\mathrm{t}}=9.6 \mathrm{~Hz}, \mathrm{~J}_{\mathrm{d}}=5.4 \mathrm{~Hz}, 1 \mathrm{H}\right) ; 2.75\left(\mathrm{dd}, \mathrm{J}_{1}=14.4 \mathrm{~Hz}, \mathrm{~J}_{2}=4.8 \mathrm{~Hz}, 1 \mathrm{H}\right) ; 2.45$ (dd, $\mathrm{J}_{1}=14.4 \mathrm{~Hz}$ ， $\left.\mathrm{J}_{2}=8.4 \mathrm{~Hz}, 1 \mathrm{H}\right) ; 2.35-1.72(\mathrm{~m}, 10 \mathrm{H}) ; 1.60-1.10(\mathrm{~m}, 6 \mathrm{H}) ; 1.43(\mathrm{~s}, 9 \mathrm{H}) ; 1.29(\mathrm{~s}, 3 \mathrm{H})$; $1.19(\mathrm{~s}, 2 \mathrm{H}) ; 1.02(\mathrm{t}, \mathrm{J}=7.8 \mathrm{~Hz}, 9 \mathrm{H}) ; 0.59(\mathrm{q}, \mathrm{J}=7.8 \mathrm{~Hz}, 6 \mathrm{H}) .{ }^{13} \mathrm{C} \mathrm{NMR}\left(75 \mathrm{MHz}, \mathrm{C}_{6} \mathrm{D}_{6}\right)$; TM $(\mathrm{ppm}): 170.1,111.1,108.2,79.8,73.7,70.3,67.9,61.4,42.5,40.6,38.2,34.7$, $31.5,31.2,29.8,28.2,24.7,20.5,18.4,12.5,7.4,7.2$. HRMS (ESI) calcd for $\mathrm{C}_{36} \mathrm{H}_{70} \mathrm{O}_{7} \mathrm{Si}_{2} \mathrm{Na} \quad[\mathrm{M}+\mathrm{Na}] 693.4558$, found 693.4564 .

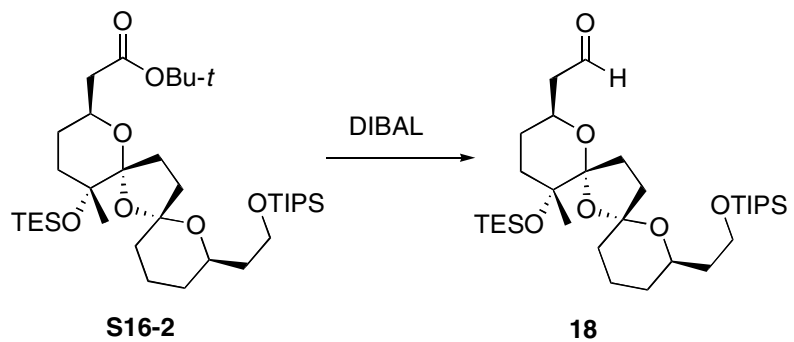

Aldehyde 18: To a solution of the TES-ether s16-2 (61.5 mg, 0.0916 mmol) in dichloromethane (1.5 mL) was slowly added DIBAL-H solution (1 M in toluene, $0.10 \mathrm{~mL}$, $0.10 \mathrm{mmol})$ at $-78{ }^{\circ} \mathrm{C}$. The reaction mixture was stirred for $1 \mathrm{~h}$ at $-78{ }^{\circ} \mathrm{C}$ and then quenched with Rochelle's solution at $-78^{\circ} \mathrm{C}$. The mixture was allowed to warm to room temperature and stirred for $30 \mathrm{~min}$ and then extracted with ethyl acetate. The combined organic layers were dried over $\mathrm{MgSO}_{4}$, filtered and concentrated. The residue was purified by flash column chromatography (silica, 5\% ethyl acetate-hexanes) to yield aldehyde $18(48.3 \mathrm{mg}, 0.0806 \mathrm{mmol}, 88 \%) .\left[\langle]_{\mathrm{D}}{ }^{23}-19.2^{\circ}\left(\mathrm{C} 1.0, \mathrm{CH}_{2} \mathrm{Cl}{ }_{2}\right) ;{ }^{1} \mathrm{H} \mathrm{NMR}\right.$ $\left(300 \mathrm{MHz}, \mathrm{C}_{6} \mathrm{D}_{6}\right) ; \mathrm{TM}(\mathrm{ppm}): 9.88\left(\mathrm{dd}, \mathrm{J}_{1}=3.0 \mathrm{~Hz}, \mathrm{~J}_{2}=1.8 \mathrm{~Hz}, 1 \mathrm{H}\right) ; 4.51-4.41$ (m, 1H); 4.12 
(ddt, $J_{t}=6.0 \mathrm{~Hz}, J_{d 1}=11.1 \mathrm{~Hz}, J_{d 2}=1.8 \mathrm{~Hz}, 1 \mathrm{H}$ ); 3.92 (dt, $\mathrm{J}_{\mathrm{d}}=10.2 \mathrm{~Hz}, \mathrm{~J}_{\mathrm{t}}=7.2 \mathrm{~Hz}, 1 \mathrm{H}$ ) ; 3.79 (ddd, $\mathrm{J}_{1}=10.2 \mathrm{~Hz}, \mathrm{~J}_{2}=7.2 \mathrm{~Hz}, \mathrm{~J}_{3}=6.0 \mathrm{~Hz}, 1 \mathrm{H}$ ); 2.36 (ddd, $\mathrm{J}_{1}=16.2 \mathrm{~Hz}, \mathrm{~J}_{2}=8.4 \mathrm{~Hz}$, $\left.\mathrm{J}_{3}=3.0 \mathrm{~Hz}, 1 \mathrm{H}\right) ; 2.26-1.05(\mathrm{~m}, 17 \mathrm{H}) ; 1.23(\mathrm{~s}, 3 \mathrm{H}) ; 1.13(\mathrm{~s}, 21 \mathrm{H}) ; 1.02$ (t, J=7.8 Hz， $9 \mathrm{H}) ; 0.58(\mathrm{q}, \mathrm{J}=7.8 \mathrm{~Hz}, 6 \mathrm{H}) \cdot{ }^{13} \mathrm{C} \mathrm{NMR}\left(75 \mathrm{MHz}, \mathrm{C}_{6} \mathrm{D}_{6}\right) ;{ }^{\mathrm{TM}}(\mathrm{ppm}): 200.2,110.7,108.4,73.3$, $70.3,66.2,60.8,49.3,40.4,38.0,34.8,34.7,31.1,30.9,30.1,24.6,20.3,18.3$, $12.4,7.4,7.2$. HRMS (ESI) calcd for $\mathrm{C}_{32} \mathrm{H}_{62} \mathrm{O}_{6} \mathrm{Si}_{2} \mathrm{Na}$ [M+Na] 621.3983, found 621.3956 . 